\title{
Highest response ratio next (HRRN) vs first come first served (FCFS) scheduling algorithm in grid environment
}

\begin{abstract}
Research on Grid scheduling nowadays, focuses in solving three problems such as finding a good algorithm, automating the process, and building a flexible, scalable, and efficient scheduling mechanism. The complexity of scheduling predicament increases linearly with the size of the Grid. Submitted jobs in Grid environment are put in queue due to the large number of jobs submission. An adequate Grid scheduling technique used to schedule these jobs and sending them to their assigned resources. This paper simulates in $\mathrm{C}$ programming First Come First Served (FCFS) and Highest Response Ratio Next (HRRN) Grid scheduling algorithms. A good scheduling algorithm normally shows lower value of total waiting and schedule time. Hence, HRRN was selected because of the algorithm outperform the existing gLite Grid middleware scheduling. From the simulation result proof HRRN has better performance of total waiting time due to the priority scheme policy implementation in scheduler.
\end{abstract}

Keyword: Grid computing; Scheduling; Simulation; Highest response ration next algorithm 